\title{
An axiomatic approach to egalitarianism in TU-games
}

\author{
Javier Arin · Jeroen Kuipers • Dries Vermeulen
}

Accepted: 19 May 2008 / Published online: 2 July 2008

(C) The Author(s) 2008

\begin{abstract}
A core concept is a solution concept on the class of balanced games that exclusively selects core allocations. We show that every continuous core concept that satisfies both the equal treatment property and a new property called independence of irrelevant core allocations (IIC) necessarily selects egalitarian allocations. IIC requires that, if the core concept selects a certain core allocation for a given game, and this allocation is still a core allocation for a new game with a core that is contained in the core of the first game, then the core concept also chooses this allocation as the solution
\end{abstract}

Javier Arin gratefully acknowledges the financial support by EHU, project nr,

UPV 035.321-HB048/97 and by the Spanish Ministry of Education and Science, project nr, SEJ2006-05455.

Jeroen Kuipers gratefully acknowledges the financial support by the Basque Government,

Department of Research and Education.

Dries Vermeulen gratefully acknowledges the financial support of the Dutch Organization for Scientific Research NWO.

We thank two referees, an associate editor and Peter Sudhölter for their remarks that helped to improve the presentation of this article considerably.

\section{J. Arin}

Departamento Fundamentos del Análisis Económico I,

Universidad del País Vasco - EHU Fac. de Ciencias Económicas y Empresariales Avda,

Lehendakari Agirre 83, 48015 Bilbao, Spain

e-mail: jeparagj@bs.ehu.es

\section{J. Kuipers}

Department of Mathematics, University of Maastricht, PO Box 616, 6200 MD Maastricht, The Netherlands e-mail: kuipers@math.unimaas.nl

D. Vermeulen $(\bowtie)$

Department of Quantitative Economics, University of Maastricht, PO Box 616, 6200 MD Maastricht, The Netherlands

e-mail: d.vermeulen@ke.unimaas.nl 
to the new game. When we replace the continuity requirement by a weak version of additivity we obtain an axiomatization of the egalitarian solution concept that assigns to each balanced game the core allocation minimizing the Euclidean distance to the equal share allocation.

Keywords TU-game $\cdot$ Egalitarianism $\cdot$ Axiomatization

\section{Introduction}

Egalitarianism is the strife of a community to spread the total wealth of the community as equally as possible among its members, while satisfying certain stability requirements of the allocation. The notion of egalitarianism is frequently used outside the theory of transferable utility games. See for example Thomson (1994) for applications in bargaining theory and Moulin (1988) for applications in welfare economics.

Dutta and Ray (1989) first introduced egalitarianism ${ }^{1}$ for TU-games. They showed that, for their definition of an egalitarian allocation, a TU-game can have at most one such allocation. They also showed that for convex games the egalitarian allocation always exists and that it is an element of the core. On the other hand, they present two examples, one showing that the egalitarian allocation may exist for games with an empty core and another one showing that the egalitarian allocation need not be a core element even if the core is not empty.

Arin and Iñarra (2001) used another definition of egalitarian allocations. The difference does not lie in the notion of egalitarianism, ${ }^{2}$ but in a the notion of stability they use. Dutta and Ray (1989) define stability of a given allocation recursively, by requiring that no coalition can object to it using an allocation that is both stable with respect to the coalition and better for all of its members. Arin and Iñarra (2001) require that the allocation be in the core of the game. As a consequence, the latter type of egalitarian allocation exists for a given game precisely when the core of the game is not empty. Arin and Iñarra (2001) showed that their definition coincides with the definition in Dutta and Ray (1989) on the class of convex games. This, together with the guarantee of existence for a relatively large and manageable class of games, makes the notion of Arin and Iñarra an interesting alternative for the notion of Dutta and Ray. The most prominent drawback of the definition of Arin and Iñarra is that more than one allocation may be egalitarian. For this reason we will introduce, in Sect. 3, several solution concepts that assign exactly one egalitarian allocation (according to the definition of Arin and Iñarra) to each balanced game.

An objective way to support the rationale of a certain solution concept (no matter whether it is single valued or set valued) is through axiomatizations. For example, the solution concept of Dutta and Ray has been axiomatized on the class of convex games, by Dutta (1990); Klijn et al. (1998). In this paper, we aim for the axiomatiza-

\footnotetext{
1 In Dutta and Ray (1991) the authors present an alternative version of their definition in the paper of (1989).

2 Both Arin and Iñarra's paper and Dutta and Ray's paper use the widely accepted Lorenz criterion as a measure for egalitarianism.
} 
tion of egalitarian solution concepts on the larger class of balanced games. While the axiomatizations of the Dutta and Ray solution are all based on some form of consistency (both Hart-Mas-Colell and Maschler-Davis reduced games can be used, even both at once), we will use an axiom that is closely related to the property known as independence of irrelevant alternatives. The connection of this property with egalitarianism has already been established outside the context of TU-games. More precisely, we will show, in Sect. 4 , that any continuous core concept ${ }^{3}$ that satisfies the equal treatment property and a property we called independence of irrelevant core allocations (IIC) must necessarily be egalitarian. By replacing continuity with a weak version of additivity, ${ }^{4}$ we obtain, in Sect. 5, an axiomatization of the egalitarian solution concept known as the least squares solution concept.

\section{Preliminaries}

Let $v: 2^{N} \rightarrow \mathbb{R}$ be a TU-game on the player set $N=\{1, \ldots, n\}$. We assume $n \geq 2$ throughout. The central problem is how to allocate the total gain $v(N)$ among the players.

An allocation is a vector $x \in \mathbb{R}^{N}$. The $i$ th coordinate $x_{i}$ of the allocation $x$ represents the payoff to player $i \in N$. For any subset of players $S \subseteq N$, their aggregate payoff $\sum_{i \in S} x_{i}$ is denoted by $x(S)$. An allocation $x$ is efficient if it distributes the worth of the grand coalition among the players, i.e. if $x(N)=v(N)$. An efficient allocation $x$ is called a core allocation if

$$
x(S) \geq v(S) \quad \text { for all } S \subseteq N .
$$

The set $C(v)$ of core allocations is called the core of the game $v$. We restrict attention to games with a non-empty core, that is, to balanced games (see Bondareva 1963; Shapley 1967).

For two players $k$ and $l$ in a game $v$, an efficient allocation $x$, and a real number $\alpha>0$, we say that $(k, l, x, \alpha)$ is an equalizing bilateral transfer (of size $\alpha$ from $k$ to $l$ with respect to $x$ ) if

$$
x_{k}-\alpha \geq x_{l}+\alpha .
$$

A core allocation $x$ is egalitarian if no core allocation $y$ is the result of an equalizing bilateral transfer with respect to $x$. A core allocation $x$ is strongly egalitarian if no core allocation $y$ is the result of a finite sequence of equalizing bilateral transfers starting from $x$.

For both notions we will frequently use alternative descriptions. We will briefly explain these. A coalition $S$ is said to be tight at an allocation $x$ if

$$
x(S)=v(S) .
$$

\footnotetext{
3 A core concept is a solution concept that exclusively assigns core allocations.

4 In Sect. 5, we will show that neither linearity nor its usual substitutes additivity and covariance are compatible with egalitarianism.
} 
The collection of all tight coalitions at $x$ is denoted by $\mathcal{T}(v, x)$. A core allocation $x$ is egalitarian if and only if for any pair of players $k$ and $l$ with $x_{k}>x_{l}$ there exists a coalition $S \in \mathcal{T}(v, x)$ with $k \in S$ and $l \notin S$.

The alternative description of strong egalitarianism is a bit more complicated. Let $x$ be an allocation in $\mathbb{R}^{n}$. By $\bar{x}$ we denote the vector that results from $x$ by permuting the coordinates of $x$ in such a way that $\bar{x}_{1} \geq \bar{x}_{2} \geq \cdots \geq \bar{x}_{n}$. We say that the vector $x$ Lorenz dominates the vector $y$ if

$$
\sum_{i=1}^{k} \bar{x}_{i} \leq \sum_{i=1}^{k} \bar{y}_{i} \text { for all } k \in\{1, \ldots, n\}
$$

and if at least one of these inequalities is strict. Now, Lemma 2 of Hardy et al. (1952) implies that a core allocation $y$ Lorenz dominates another core allocation $x$ only if $y$ can be obtained from $x$ by a finite sequence of equalizing bilateral transfers. Hence, since the converse of this Lemma is trivial, a core element is strongly egalitarian if and only if it is undominated in the sense of Lorenz. We use this characterization of strong egalitarianism in terms of the Lorenz criterion freely throughout the paper. We also frequently use the term Lorenz allocation for a strongly egalitarian core allocation.

Observe that the equal share allocation $\operatorname{Es}(v)$ (the allocation that allocates $\frac{v(N)}{|N|}$ to each player) is the unique Lorenz allocation whenever it is a core element. If $\operatorname{Es}(v)$ is not in the core of $v$, then there may be more Lorenz allocations as the next example shows.

Example 1 Consider the four player game $v$ defined by

$$
v(S):=\left\{\begin{aligned}
7 & \text { if } S=\{1,2\} \text { or }\{1,3\} \\
12 & \text { if } S=\{1,2,3,4\} \\
0 & \text { else. }
\end{aligned}\right.
$$

Note that $v(S \cup\{1\}) \geq v(S \cup\{2\})$ for all $S \subseteq N \backslash\{1,2\}$. It follows that an equalizing transfer from 2 to 1 is possible if $x_{1}<x_{2}$ for some core element $x$. Hence, any egalitarian allocation $x$ must satisfy $x_{1} \geq x_{2}$. We also have $x_{2}=x_{3}$, since $v(S \cup\{2\})=$ $v(S \cup\{3\})$ for all $S \subseteq N \backslash\{2,3\}$, and $x_{3} \geq x_{4}$ since $v(S \cup\{3\}) \geq v(S \cup\{4\})$ for all $S \subseteq N \backslash\{3,4\}$. Moreover, $x_{1}>x_{4}$, since the equal share allocation is not in the core. Therefore, $x_{1}+x_{2}=x_{1}+x_{3}=7$, since an equalizing transfer from player 1 to player 4 would be possible in case $x_{1}+x_{2}=x_{1}+x_{3}>7$. We conclude that all egalitarian allocations are of the form

$$
x=(7-\lambda, \lambda, \lambda, 5-\lambda)
$$

with $2 \frac{1}{2} \leq \lambda \leq 3 \frac{1}{2}$. Finally, observe that none of these allocations Lorenz dominates any of the other allocations. Hence, these are indeed also all Lorenz allocations. 


\section{Some egalitarian concepts}

Since a balanced game may have more than one core allocation, it makes sense to study solution concepts that select precisely one core allocation for each balanced game. We call such a solution concept a core concept. We say that a core concept is an egalitarian concept if it assigns only egalitarian core allocations. It is called a Lorenz concept if it exclusively assigns Lorenz allocations.

Definition 1 For a balanced game $v$, the least squares solution $\operatorname{LS}(v)$ is defined as the unique allocation $x$ in $C(v)$ for which $\|x\| \leq\|y\|$ for all $y \in C(v)$. Here, $\|x\|$ denotes the Euclidean length $\sqrt{\sum_{i \in N} x_{i}^{2}}$ of $x$.

Notice that LS is indeed a Lorenz concept, since the value of $\|\cdot\|$ decreases whenever an equalizing bilateral transfer is made. A useful geometric interpretation of $\operatorname{LS}(v)$ is that it is the unique point in $C(v)$ that minimizes the Euclidean distance to Es $(v)$, the ideal, or maybe better, ultimate, egalitarian allocation.

The LS concept is not the only egalitarian core concept. For example, Arin and Iñarra (2001) introduced the lexmax and the lexmin concept. The definition of these concepts is as follows. An allocation $x$ said to be preferred to another allocation $y$ if $\bar{x} \neq \bar{y}$ and if $\bar{x}_{i}<\bar{y}_{i}$ for the first coordinate $i$ with $\bar{x}_{i} \neq \bar{y}_{i}$, i.e. if the vector $\bar{x}$ is lexicographically smaller than $\bar{y}$. This is denoted by $\bar{x} \prec_{\text {lex }} \bar{y}$. By $\bar{x} \preceq_{\text {lex }} \bar{y}$ we mean $\bar{x}=\bar{y}$ or $\bar{x} \prec_{\text {lex }} \bar{y}$.

Definition 2 For a balanced game $v$, the lexmax solution $\operatorname{Lmax}(v)$ is defined as the set of most preferred core allocations, i.e.

$$
\operatorname{Lmax}(v)=\left\{x \in C(v) \mid \bar{x} \preceq_{\text {lex }} \bar{y} \text { for all } y \in C(v)\right\} .
$$

Similarly, the lexmin solution $\operatorname{Lmin}(v)$ is defined as

$$
\operatorname{Lmin}(v)=\left\{x \in C(v) \mid \overline{-x} \preceq_{l e x} \overline{-y} \text { for all } y \in C(v)\right\} .
$$

It is easy to prove that allocations in $\operatorname{Lmax}(v)$ and $\operatorname{Lmin}(v)$ are Lorenz allocations, and it follows from Lemma 1.1 in Moulin (1988) that $\operatorname{Lmax}(v)$ and $\operatorname{Lmin}(v)$ consist of a unique core allocation for every game $v$. Therefore, we may interpret Lmax and Lmin as Lorenz concepts and we will abuse notation slightly by writing e.g. $x=\operatorname{Lmax}(v)$ instead of $x \in \operatorname{Lmax}(v)$. In the following, we will provide a proof for the continuity of the Lmax concept. A similar proof can be given for Lmin. The proof closely resembles the proof of Kohlberg (1971) for uniqueness and continuity of the nucleolus. ${ }^{5}$ Also, a similar proof for the continuity of the set of all Lorenz allocations was given in Hougaard et al. (2001).

Definition 3 Let $\mathcal{I}=\left(I_{1}, \ldots, I_{p}\right)$ be an ordered partition of $N$ and let $\mathcal{T}$ be a collection of subsets of $N$. We say that the pair $(\mathcal{I}, \mathcal{T})$ has property $\mathbf{I}$ if $z=0$ is the unique solution to the following system of (in)equalities.

\footnotetext{
5 This type of proof was suggested to us by one of the referees.
} 
(1) $z(N)=0$,

(2) $z(S) \geq 0$ for all $S \in \mathcal{T}$,

(3) For every $k \in\{1, \ldots, p\}$ : If $z_{i}=0$ for all $i \in \bigcup_{\ell<k} I_{\ell}$, then $z_{i} \leq 0$ for all $i \in I_{k}$.

Definition 4 Given an allocation $x$, we define

$$
I_{1}(x)=\left\{i \in N \mid x_{i} \geq x_{j} \text { for all } j \in N\right\}
$$

and for $k \geq 2$ we define $I_{k}(x) \subseteq N$ recursively by

$$
I_{k}(x)=\left\{i \notin \bigcup_{\ell<k} I_{\ell}(x) \mid x_{i} \geq x_{j} \quad \text { for all } j \notin \bigcup_{\ell<k} I_{\ell}(x)\right\}
$$

Take $p=\max \left\{k \mid I_{k}(x) \neq \emptyset\right\}$. The vector $\mathcal{I}(x)=\left(I_{1}(x), \ldots, I_{p}(x)\right)$ of coalitions is called the ordered partition associated with $x$. Using this terminology, we get the following characterization of the $\operatorname{Lmax}(v)$ allocation.

Lemma 1 Let $v$ be a balanced game and let $x \in C(v)$. Then

$$
x=\operatorname{Lmax}(v) \Leftrightarrow \text { the pair }(\mathcal{I}(x), \mathcal{T}(v, x)) \text { has property } \mathbf{I} \text {. }
$$

Proof Necessity: suppose that $(\mathcal{I}(x), \mathcal{T}(v, x))$ does not have property $\mathbf{I}$. Then there exists a $z \neq 0$ that satisfies conditions (1) till (3). Let

$$
k=\min \left\{\ell \mid \text { there is an } i \in I_{\ell}(x) \text { with } z_{i} \neq 0\right\}
$$

Let $i \in I_{k}(x)$ such that $z_{i} \neq 0$. Then $z_{i}<0$ by (3). Therefore $\overline{x+\epsilon z} \prec_{\text {lex }} \bar{x}$ for sufficiently small $\epsilon>0$. Also, by (1) and (2), $\overline{x+\epsilon z} \in C(v)$ for $\epsilon>0$ sufficiently small. Hence, $x$ cannot be equal to $\operatorname{Lmax}(v)$.

Sufficiency: Suppose $(\mathcal{I}(x), \mathcal{T}(v, x))$ has property $\mathbf{I}$. Let $y \in C(v)$ such that $\bar{y} \preceq_{\text {lex }}$ $\bar{x}$ and let $z=y-x$. We show that $z=0$. Clearly $z(N)=y(N)-x(N)=v(N)-$ $v(N)=0$, which shows (1). Further, since $y(S) \geq v(S)$ for all $S \subseteq N, z(S)=$ $y(S)-x(S)=y(S)-v(S) \geq 0$ for all $S \in \mathcal{T}(v, x)$, which shows (2). To prove (3), let $k \in\{1, \ldots, p\}$ and assume

$$
z_{i}=0 \quad \text { for all } i \in \bigcup_{\ell<k} I_{\ell}(x)
$$

Let $i^{*} \in I_{k}(x)$. It remains to show that $z_{i} i \leq 0$. Without loss of generality we can assume that $\bar{x}=x$ and $y_{i} \geq y_{j}$ for all $i, j \in I_{k}(x)$ with $i \leq j$. (This can be achieved by permuting the player set if necessary.) Since $y \preceq_{l e x} \bar{y} \preceq_{l e x} \bar{x}=x, z_{i} \leq^{*} 0$ by Eq. (*).

Before we go on to prove that Lmax is continuous, we introduce the following terminology. We say that $(\mathcal{J}, \mathcal{S})$ is derived from $(\mathcal{I}, \mathcal{T})$ if $\mathcal{T} \subseteq \mathcal{S}$ and if $J_{1}=$ $I_{1} \cup \cdots \cup I_{k_{1}}, J_{2}=I_{k_{1}+1} \cup \cdots \cup I_{k_{2}}$, etc. It is easy to see that, if $(\mathcal{I}, \mathcal{T})$ has property $\mathbf{I}$, then also $(\mathcal{J}, \mathcal{S})$ has property $\mathbf{I}$ if it is derived from $(\mathcal{I}, \mathcal{T})$. 
Theorem 1 The core concept Lmax is continuous.

Proof Let $\left(v^{k}\right)_{k \in \mathbb{N}}$ be a sequence of balanced games converging to $v$. Denote, for every $k \in \mathbb{N}, \operatorname{Lmax}\left(v^{k}\right)$ by $x^{k}$. Since the core correspondence is compact valued and continuous, we know that the sequence $\left(x^{k}\right)_{k \in \mathbb{N}}$ is bounded. Thus, we have to show that every convergent subsequence of $\left(x^{k}\right)_{k \in \mathbb{N}}$ converges to Lmax $(x)$. Assume without loss of generality that $\left(x^{k}\right)_{k \in \mathbb{N}}$ converges to $x$. Consider the sequence $\left(\mathcal{I}\left(x^{k}\right), \mathcal{T}\left(v^{k}, x^{k}\right)\right)_{k \in \mathbb{N}}$. Since there are only finitely ordered partitions and only finitely many collections of coalitions, we can assume without loss of generality that there is a pair $(\mathcal{I}, \mathcal{T})$ such that $\left(\mathcal{I}\left(x^{k}\right), \mathcal{T}\left(v^{k}, x^{k}\right)\right)=(\mathcal{I}, \mathcal{T})$ for all $k \in \mathbb{N}$. By Lemma $1,(\mathcal{I}, \mathcal{T})$ has property I. Since all weak inequalities are preserved in passing to the limit, it follows that $(\mathcal{I}(x), \mathcal{T}(v, x))$ is derived from $(\mathcal{I}, \mathcal{T})$. We conclude that $(\mathcal{I}(x), \mathcal{T}(v, x))$ has property $\mathbf{I}$ as well. By Lemma 1, $x=\operatorname{Lmax}(v)$.

The LS solution minimizes a continuous and strictly convex function over the core of the game. Since the core is compact-valued and continuous, it follows immediately that LS is also continuous. Therefore, all three concepts Lmax, Lmin and LS are continuous $(\mathrm{CON})$.

We now introduce another property that is also shared by all three of the solutions LS, Lmax and Lmin. Informally, this property states that a chosen core allocation should not be discarded if it is still available in a smaller core. The interpretation of this is that only "irrelevant" core allocations have disappeared, hence there is no reason to change. A related property, called independence of irrelevant alternatives, is widely used in the context of bargaining situations. Formally, a core concept $\phi$ is called independent of irrelevant core allocations (IIC) if $\phi(v)=\phi(w)$ for any two games $v$ and $w$ with $\phi(v) \in C(w) \subseteq C(v)$. Since LS, Lmax and Lmin all maximize some preference relation on $\mathbb{R}^{N}$ over the core, it is immediate that all three core concepts satisfy IIC.

Say that two players $i$ and $j$ are symmetric in a game $v$ if $v(S \cup\{i\})=v(S \cup\{j\})$ for all $S \subseteq N \backslash\{i, j\}$. Say that a core concept $\phi$ satisfies the equal treatment property (ETP) if $\phi_{i}(v)=\phi_{j}(v)$ whenever $i$ and $j$ are symmetric in $v$. It is trivial that LS, Lmax and Lmin all satisfy ETP.

\section{Sufficient conditions for egalitarianism}

We have seen that the Lorenz concepts Lmax, Lmin and LS all satisfy IIC, ETP and CON. Hence, these three properties do not single out a unique core concept when $n \geq 4 .{ }^{6}$ It is also not true that every egalitarian solution concept satisfies IIC or CON (egalitarian solution concepts always satisfy ETP). ${ }^{7}$ We show however that every core

\footnotetext{
$\overline{6}$ For the game $v$ in Example 1 , one can check that $\operatorname{Lmax}(v)=\left(3 \frac{1}{2}, 3 \frac{1}{2}, 3 \frac{1}{2}, 1 \frac{1}{2}\right), \operatorname{Lmin}(v)=$ $\left(4 \frac{1}{2}, 2 \frac{1}{2}, 2 \frac{1}{2}, 2 \frac{1}{2}\right)$, and $\operatorname{LS}(v)=(4,3,3,2)$ (since for $\lambda=3$ the squared distance of an arbitrary Lorenz point $(7-\lambda, \lambda, \lambda, 5-\lambda)$ to the origin, i.e. $(7-\lambda)^{2}+2 \lambda^{2}+(5-\lambda)^{2}$, is minimized). So the concepts are indeed different.

7 Using Example 1 it is straightforward to construct an egalitarian concept that is not continuous.
} 
concept satisfying IIC, ETP and CON is egalitarian. We will first prove this statement for a special class of games.

Lemma 2 Let $\phi$ be a core concept that satisfies ETP and IIC. Let $v$ be a game for which the equal share allocation $E s(v)$ is an element of the core of $v$. Then $\phi(v)=E s(v)$.

Proof Let $v$ be a game such that $\operatorname{Es}(v)$ is an element of the core of $v$. Define $m:=$ $\min \{v(S) \mid S \subseteq N\}$ and define the game $w$ by

$$
w(S):= \begin{cases}m & \text { if } S \neq N \\ v(N) & \text { if } S=N\end{cases}
$$

Since $w \leq v$ and $w(N)=v(N)$, we have $\operatorname{Es}(v) \in C(v) \subseteq C(w)$. Hence, $w$ is a balanced game and it immediately follows from ETP that $\phi(w)=\operatorname{Es}(v)$. Applying IIC we also find that $\phi(v)$ equals $\operatorname{Es}(v)$.

For a core concept that satisfies IIC, the solution is not discarded if the core shrinks and the solution stays available, by definition. If the core concept is also continuous, then the solution is not discarded even if the core grows, provided that the growth is not in the neighborhood of the solution. The following lemma formalizes this statement.

Lemma 3 Let $\phi$ be a core concept satisfying IIC and CON. Let $v$ and $w$ be two balanced games such that $\phi(v) \in C(w)$, and $w(S)=v(S)$ for every $S \in \mathcal{T}(v, \phi(v))$. Then $\phi(w)=\phi(v)$.

Proof Let $\phi, v$ and $w$ be as described above. For every $\lambda \in[0,1]$ define the (balanced) game $u(\lambda)$ by $u(\lambda):=\lambda w+(1-\lambda) v$. Define

$$
\bar{\lambda}:=\max \{\lambda \mid \phi(u(\lambda))=\phi(v)\} .
$$

This maximum exists, since $\phi$ is continuous. Clearly we only need to show that $\bar{\lambda}=1$. Suppose that $\bar{\lambda}<1$. We derive a contradiction. Consider a coalition $S$ for which $w(S) \neq v(S)$. By assumption $\phi(v)(S)>v(S)$, and hence

$$
\phi(u(\bar{\lambda}))(S)=\phi(v)(S)>v(S) .
$$

Since $\phi$ is continuous and there are only finitely many coalitions $S$ with $w(S) \neq v(S)$, we can find a number $\mu>\bar{\lambda}$ such that we still have $\phi(u(\mu))(S)>v(S)$ for all such coalitions $S$. Define the game $u^{*}$ by, for all $S, u^{*}(S)=\max \{u(\mu)(S), v(S)\}$. Since for all remaining coalitions $S$, the ones with $w(S)=v(S)$, we have

$$
\phi(u(\mu))(S) \geq u(\mu)(S)=\mu w(S)+(1-\mu) v(S)=v(S)
$$

it follows that $\phi(u(\mu)) \in C\left(u^{*}\right)$. However, also $\phi(v) \in C\left(u^{*}\right)$ since $\phi(v) \in C(u(\lambda))$ for all $\lambda$ by assumption. Therefore, since both $u^{*} \geq v$ and $u^{*} \geq u(\mu), \phi(v)=\phi\left(u^{*}\right)=$ $\phi(u(\mu))$ by IIC. Contradiction.

Now, we can prove the main result of this section. 
Theorem 2 Every core concept on the class of balanced games that satisfies IIC, ETP and $C O N$ is egalitarian.

Proof Let $\phi$ be a core concept that satisfies the axioms IIC, ETP and CON, and let $v$ be a balanced game. Assume that we have two players $k$ and $l$ such that $\phi(v)_{k}>\phi(v)_{l}$. By the alternative description of egalitarianism we have to show that there is a coalition $S$ with $k \in S$ and $l \notin S$ such that $\phi(v)(S)=v(S)$.

Let $\mathcal{B}$ denote the collection of coalitions $S$ with $k \in S$ and $l \notin S$. Assume conversely that $\phi(v)(S)>v(S)$ for every $S \in \mathcal{B}$. We will derive a contradiction by showing that $\phi(v)_{k}=\phi(v)_{l}$. Construct the auxiliary game $w$ as follows.

$$
w(S):= \begin{cases}v((S \cup\{l\}) \backslash\{k\}) & \text { if } S \in \mathcal{B} \\ v(S) & \text { if } S \notin \mathcal{B} .\end{cases}
$$

We first show that $\phi(v) \in C(w)$. Clearly $\phi(v)(S) \geq v(S)=w(S)$ for $S \notin \mathcal{B}$. For $S \in \mathcal{B}$,

$$
\phi(v)(S)>\phi(v)((S \cup\{l\}) \backslash\{k\}) \geq v((S \cup\{l\}) \backslash\{k\})=w(S) .
$$

Now take a coalition $S$ with $w(S) \neq v(S)$. Necessarily $S \in \mathcal{B}$. Thus $\phi(v)(S)>v(S)$ by assumption. It follows from Lemma 3 that $\phi(v)=\phi(w)$.

Finally notice that players $k$ and $l$ are symmetric in $w$. Therefore $\phi(v)_{k}=\phi(w)_{k}=$ $\phi(w)_{l}=\phi(v)_{l}$ by ETP. Contradiction.

We show that all three requirements in Theorem 2 are necessary to ensure egalitarianism. To see that ETP and CON are not sufficient, notice that the nucleolus satisfies these two properties, while it is not egalitarian. Let $\phi$ denote the core concept that assigns the lexicographically maximal vector in $C(v)$ to any balanced game $v$. (It is indeed a core concept, since the core is compact and convex, hence the lexicographically maximal vector in $C(v)$ is uniquely determined.) Then $\phi$ is IIC, and it also satisfies CON, which can be shown using an appropriately adapted version ${ }^{8}$ of the proof of the continuity of Lmax. Clearly, $\phi$ is not egalitarian, which shows that IIC and $\mathrm{CON}$ are not sufficient to ensure egalitarianism.

Also, the requirements of ETP and IIC are not enough to ensure that a core concept is egalitarian on the class of balanced games, as the following example shows. For any balanced TU game $v$, define $M_{1}(v)$ as the set of players with lowest payoff in $\operatorname{Lmax}(v)$ and $M_{2}(v)$ as the set of players with second-lowest payoff in $\operatorname{Lmax}(v)$. We define $\psi(v)=\operatorname{Lmax}(v)$, unless $M_{1}(v)$ and $M_{2}(v)$ both consist of exactly one player. In this case, let $k$ denote the unique player in $M_{1}(v)$ and let $l$ denote the unique player in $M_{2}(v)$. Write

$$
x(\lambda)=\operatorname{Lmax}(v)+\lambda\left(e_{l}-e_{k}\right)
$$

define

$$
\lambda^{*}=\max \left\{\lambda \mid x(\lambda) \in C(v) \text { and } x(\lambda)_{l} \leq \operatorname{Lmax}(v)_{i} \text { for all } i \neq k, l\right\},
$$

and define $\psi(v)=x\left(\lambda^{*}\right)$. Clearly, $\psi$ is a core concept.

\footnotetext{
8 In fact the proof is somewhat easier because the lexicographic ordering is applied directly to core elements $x$ instead of to the ordered vector $\bar{x}$.
} 
Proposition 1 The core concept $\psi$ satisfies ETP and IIC.

Proof To see that $\psi$ satisfies ETP, suppose that players $i$ and $j$ are symmetric in $v$. Since Lmax satisfies ETP, it is trivial that $\psi(v)_{i}=\psi(v)_{j}$, whenever $\psi(v)=\operatorname{Lmax}(v)$. Now consider the case that there is a unique player $k$ in $M_{1}(v)$ and a unique player $l$ in $M_{2}(v)$. Since Lmax satisfies ETP, it follows that $\{i, j\}$ has an empty intersection with $\{k, l\}$. Therefore,

$$
\psi(v)_{i}=\operatorname{Lmax}(v)_{i}=\operatorname{Lmax}(v)_{j}=\psi(v)_{j}
$$

Next, we show that $\psi$ satisfies IIC. Suppose that games $v$ and $w$ are such that $\psi(v) \in$ $C(w) \subseteq C(v)$. We show that $\psi(w)=\psi(v)$. First consider the case that $M_{1}(v)$ and $M_{2}(v)$ are not both singleton sets. Then $\psi(v)=\operatorname{Lmax}(v)$, hence $\operatorname{Lmax}(v) \in C(w)$. It follows that $\operatorname{Lmax}(w)=\operatorname{Lmax}(v)$. Then trivially, $M_{1}(w)$ and $M_{2}(w)$ are not both singleton sets, hence $\psi(w)=\operatorname{Lmax}(w)=\operatorname{Lmax}(v)=\psi(v)$.

Now assume that $k$ is the unique player in $M_{1}(v)$ and $l$ is the unique player in $M_{2}(v)$. Since $C(w) \subseteq C(v)$, we have $\overline{\operatorname{Lmax}(v)} \preceq_{\text {lex }} \overline{\operatorname{Lmax}(w)}$, and since $\psi(v) \in C(w)$, we have $\overline{\operatorname{Lmax}(w)} \preceq_{l e x} \overline{\psi(v)}$. Hence,

$$
\overline{\operatorname{Lmax}(v)} \preceq_{\text {lex }} \overline{\operatorname{Lmax}(w)} \preceq_{\text {lex }} \overline{\psi(v)} .
$$

By definition of $\psi(v)$, we have $\psi(v)_{i}=\operatorname{Lmax}(v)_{i}$ for all $i \in N \backslash\{k, l\}$, i.e. $\psi(v)_{i}=$ $\operatorname{Lmax}(v)_{i}$ for the $n-2$ highest coordinates of $\operatorname{Lmax}(v)$. It follows that

$$
{\overline{\operatorname{Lmax}(v)_{i}}}_{i}={\overline{\operatorname{Lmax}(w)_{i}}}_{i}={\overline{\psi(v)_{i}}}_{i} \text { for } i=1, \ldots, n-2,
$$

and it even follows that $\operatorname{Lmax}(w)_{i}=\operatorname{Lmax}(v)_{i}$ for all $i \neq k, l$.

Thus, we have

$$
\operatorname{Lmax}(w)=\operatorname{Lmax}(v)+\hat{\mu}\left(e_{l}-e_{k}\right)
$$

for some real number $\hat{\mu}$. Note that $\hat{\mu} \geq 0$, since for $\mu<0, \operatorname{Lmax}(v)+\mu\left(e_{l}-e_{k}\right) \notin$ $C(v)$, hence $\operatorname{Lmax}(v)+\mu\left(e_{l}-e_{k}\right) \notin C(w)$. This shows that $k$ is the unique player in $M_{1}(w)$. We distinguish two cases for the number of players in $M_{2}(w)$.

A. $M_{2}(w)$ contains more than one player. This implies that $\hat{\mu}=\lambda^{*}$. It follows that $\psi(w)=\operatorname{Lmax}(w)=\operatorname{Lmax}(v)+\lambda^{*}\left(e_{l}-e_{k}\right)=\psi(v)$.

B. Player $l$ is the unique element of $M_{2}(w)$. Then $\psi(w)=y\left(\mu^{*}\right)$, where $y(\mu):=$ $\operatorname{Lmax}(w)+\mu\left(e_{l}-e_{k}\right)$ and

$$
\mu^{*}:=\max \left\{\mu \mid y(\mu) \in C(w) \text { and } y(\mu)_{l} \leq \operatorname{Lmax}(w)_{i} \text { for all } i \neq k, l\right\} .
$$

Note that $y(\mu)=x(\mu+\hat{\mu})$ for all $\mu$. Therefore, we have

$$
\begin{aligned}
\mu^{*} & =\max \left\{\mu \mid x(\mu+\hat{\mu}) \in C(w) \text { and } x(\mu+\hat{\mu})_{l} \leq \operatorname{Lmax}(v)_{i} \quad \text { for all } i \neq k, l\right\} \\
& \leq \max \left\{\mu \mid x(\mu+\hat{\mu}) \in C(v) \text { and } x(\mu+\hat{\mu})_{l} \leq \operatorname{Lmax}(v)_{i} \quad \text { for all } i \neq k, l\right\} \\
& =\lambda^{*}-\hat{\mu} .
\end{aligned}
$$


We also have $\mu^{*} \geq \lambda^{*}-\hat{\mu}$, since $y\left(\lambda^{*}-\hat{\mu}\right)=x\left(\lambda^{*}\right)=\psi(v) \in C(w)$, and $y_{l}\left(\lambda^{*}-\hat{\mu}\right)=x_{l}\left(\lambda^{*}\right) \leq \operatorname{Lmax}(v)_{i}=\operatorname{Lmax}(w)_{i}$ for all $i \neq k, l$. It follows that $\psi(w)=y\left(\mu^{*}\right)=x\left(\lambda^{*}\right)=\psi(v)$.

We verified that $\psi$ satisfies IIC and ETP. To see that it is not egalitarian, consider the game $v$ with player set $N=\{1,2, \ldots, n\}$ defined by

$$
v(S)=\left\{\begin{aligned}
-10 & \text { if } S=N \\
-10 & \text { if } S=\{1,2\} \\
-7 & \text { if } S=\{1\} \\
-4 & \text { if } S=\{2\} \\
0 & \text { else. }
\end{aligned}\right.
$$

It is easily verified that $\psi(v)_{1}=-7, \psi(v)_{2}=-3$ and $\psi(v)_{i}=0$ for all $i \in N \backslash\{1,2\}$, while the unique egalitarian allocation $x$ is defined by $x_{1}=-6, x_{2}=-4$ and $x_{i}=0$ for all $i \in N \backslash\{1,2\}$.

We could neither prove nor disprove that a core concept with the properties of Theorem 2 is also a Lorenz concept.

\section{An axiomatization of the $\mathrm{LS}$ solution concept}

In this section, we will provide an axiomatization of the least squares solution concept. This will be done by replacing the axiom of continuity by another one that is closely related to additivity. We first show that egalitarianism is incompatible with additivity itself, as well as with its usual substitutes.

Example 2 Let $v$ be the two-person game defined by $v(\{1,2\})=2$ and $v(\{1\})=$ $v(\{2\})=0$. The only egalitarian point in the core of this game is the allocation $(1,1)$. Furthermore, let $\beta$ be the additive game defined by $\beta(\{1,2\})=0, \beta(\{1\})=1$ and $\beta(\{2\})=-1$. Then the only core element, and hence the only egalitarian allocation, is $(1,-1)$. However, the only egalitarian allocation in the core of the game $v+\beta$ is the allocation $(1,1)$, which is not equal to $(1,1)+(1,-1)$. The example is easily extended to games with $n \geq 2$.

Hence, no egalitarian core concept satisfies additivity. The example also shows that no egalitarian core concept satisfies covariance under strategic equivalence, ${ }^{9}$ not even the usual weak versions thereof. One way around this problem is the use of the following weak (and admittedly technical) version of additivity. A core concept $\phi$ is conditionally additive (CADD) if

$$
\phi(v+w)=\phi(v)+\phi(w)
$$

for any two balanced games $v$ and $w$ with $\mathcal{T}(v, \phi(v))=\mathcal{T}(w, \phi(w))$. Note that, in this case, the collection of tight coalitions at $\phi(v+w)$ in the game $v+w$ equals the collection of tight coalitions at $\phi(v)$ in the game $v$.

\footnotetext{
9 Dutta and Ray (1989) have already shown that the solution they propose is not covariant. See their paper for a convincing argument why an egalitarian solution concept should not satisfy covariance.
} 
Although the condition under which we require additivity in our CADD axiom is technical, there are core concepts that are conditionally additive in a natural way, while they do not satisfy additivity. For any solution concept $\phi$ on the class of balanced games, define the core concept $P(\phi)$ as the one that assigns to a balanced game $v$ the unique allocation in $C(v)$ for which the Euclidean distance to $\phi(v)$ is minimal.

Theorem 3 If $\phi$ is an additive solution concept, then $P(\phi)$ satisfies CADD.

Proof Define $\pi=P(\phi)$ and let $v$ and $w$ be two games such that

$$
\mathcal{T}(v, \pi(v))=\mathcal{T}(w, \pi(w))=: \mathcal{T}
$$

We show that $\pi(v+w)=\pi(v)+\pi(w)$. Since it is clear that $\pi(v)+\pi(w)$ is an element of the core of the game $v+w$, it remains to show that $\pi(v)+\pi(w)$ is the (unique) element of $C(v+w)$ for which the Euclidean distance to $\phi(v+w)$ is minimal. Let $z \in C(v+w)$. It remains to show that

$$
\|z-\phi(v+w)\| \geq\|\pi(v)+\pi(w)-\phi(v+w)\| .
$$

Write $\Delta=z-\pi(v)-\pi(w)$. Let $\langle x, y\rangle$ denote the inner product of $x$ and $y$. First we show $\langle\Delta, \pi(v)-\phi(v)\rangle \geq 0$. Suppose the contrary. Since

$$
\Delta(T)=z(T)-\pi(v)(T)-\pi(w)(T) \geq v(T)+w(T)-\pi(v)(T)-\pi(w)(T)=0
$$

for all $T \in \mathcal{T}$, it follows that $\pi(v)+\lambda \Delta \in C(v)$ for sufficiently small $\lambda>0$. Moreover, from the assumption $\langle\Delta, \pi(v)-\phi(v)\rangle<0$ we obtain

$$
\begin{aligned}
\|\pi(v)+\lambda \Delta-\phi(v)\|^{2} & =\lambda^{2}\|\Delta\|^{2}+2 \lambda\langle\Delta, \pi(v)-\phi(v)\rangle+\|\pi(v)-\phi(v)\|^{2} \\
& <\|\pi(v)-\phi(v)\|^{2}
\end{aligned}
$$

for $\lambda>0$ close to zero. This contradicts the definition of $\pi(v)$ as the closest point in $C(v)$ to $\phi(v)$. Hence, $\langle\Delta, \pi(v)-\phi(v)\rangle \geq 0$. Similarly one shows that $\langle\Delta, \pi(w)-$ $\phi(w)\rangle \geq 0$, and we have $\langle\Delta, \pi(v)+\pi(w)-\phi(v+w)\rangle \geq 0$ by the additivity of $\phi$. Thus,

$$
\begin{aligned}
\|z-\phi(v+w)\|^{2}= & \|\Delta+\pi(v)+\pi(w)-\phi(v+w)\|^{2} \\
= & \|\Delta\|^{2}+2\langle\Delta, \pi(v)+\pi(w)-\phi(v+w)\rangle+\| \pi(v)+\pi(w) \\
& -\phi(v+w) \|^{2} \\
\geq & \|\pi(v)+\pi(w)-\phi(v+w)\|^{2},
\end{aligned}
$$

which completes the proof.

We already know that the LS solution satisfies IIC and ETP. It also follows from Theorem 3 that it satisfies CADD, since $\operatorname{LS}(v)=P(\operatorname{Es}(v))$, while the equal shares solution Es is clearly additive. Our objective now is to show that the LS concept is the only core concept on the class of balanced games that satisfies IIC, ETP and CADD. 
We will first show that any core concept satisfying IIC, ETP and CADD must assign the core allocation LS for a collection of specific games. Let $\mathcal{T}$ be a collection of coalitions in $N$ with $\emptyset \in \mathcal{T}$ and $N \in \mathcal{T}$. Take $T \in \mathcal{T}$ and $\kappa>0$. Define the game $w^{T, \mathcal{T}, \kappa}$ by

$$
w^{T, \mathcal{T}, \kappa}(S):= \begin{cases}|S \cap T| & \text { if } S \in \mathcal{T} \\ -\kappa & \text { else. }\end{cases}
$$

It is easy to verify that the incidence vector $e_{T}$ of coalition $T$ is an element of the core of the game $w^{T, \mathcal{T}, \kappa}$. So, $w^{T, \mathcal{T}, \kappa}$ is balanced. Note however that this allocation need not be the only core element of $w^{T, \mathcal{T}, \kappa}$. Nevertheless,

Lemma 4 Let $\phi$ be a core concept satisfying IIC, ETP and CADD, and let $\lambda \geq 0$. Then $\phi\left(\lambda w^{T, \mathcal{T}, \kappa}\right)=\lambda e_{T}$.

Proof Take $w^{T, \mathcal{T}, \kappa}$ as above, and let $\lambda \geq 0$ and $\mu>0$. Define the game $u^{*}$ by ${ }^{10}$

$$
u^{*}(S)= \begin{cases}0 & \text { if } S=\emptyset \\ \lambda|T| & \text { if } S=T \text { or } S=N \\ -\mu|N \backslash T| & \text { if } S=N \backslash T \text { and } T \neq \emptyset, N \\ -\mu|N| & \text { otherwise. }\end{cases}
$$

Note that $u^{*}$ is balanced, since $\lambda e_{T} \in C\left(u^{*}\right)$. First we show that $\phi\left(u^{*}\right)=\lambda e_{T}$. If $T=N$ or $T=\emptyset$ the equal share allocation $\lambda e_{T}$ is in the core of $u^{*}$, and $\phi\left(u^{*}\right)=\lambda e_{T}$ by Lemma 2 .

If $T \neq N$ and $T \neq \emptyset$, by ETP there exist numbers $\alpha$ and $\beta$ such that $\phi_{i}\left(u^{*}\right)=\alpha$ for all $i \in T$ and $\phi_{i}\left(u^{*}\right)=-\beta$ for all $i \in N \backslash T$. From the fact that $\phi\left(u^{*}\right)$ is a core allocation it follows that

$$
\begin{aligned}
\alpha & \geq \lambda & & \text { by the core-constraint for } T, \\
\beta & \leq \mu & & \text { by the core-constraint for } N \backslash T, \\
\alpha|T|-\beta|N \backslash T| & =\lambda|T| & & \text { by the core-constraint for } N .
\end{aligned}
$$

Equivalently,

$$
0 \leq \beta \leq \mu \quad \text { and } \quad \alpha=\lambda+\beta \frac{|N \backslash T|}{|T|}
$$

It remains to show that $\beta=0$ and that hence $\alpha=\lambda$. Suppose that $\beta>0$. We derive a contradiction. Define the game $u^{* *}$ by

$$
u^{* *}(S)= \begin{cases}0 & \text { if } S=\emptyset \\ \lambda|N \backslash T| & \text { if } S=N \\ (\lambda+\beta-\mu) \frac{|N \backslash T|^{2}}{|N|} & \text { if } S=N \backslash T \\ -\mu & \text { else. }\end{cases}
$$

$\overline{10}$ To avoid subcases $u^{*}(\emptyset)$ is defined twice when $T=\emptyset$. 
It is easily verified that

$$
\operatorname{Es}\left(u^{* *}\right)=\lambda \frac{|N \backslash T|}{|N|} e_{N} \in C\left(u^{* *}\right),
$$

Hence, by Lemma 2, we have $\phi\left(u^{* *}\right)=\operatorname{Es}\left(u^{* *}\right)$. Furthermore, it is straightforward to check that $\mathcal{T}\left(u^{*}, \phi\left(u^{*}\right)\right)=\{N, N \backslash T\}=\mathcal{T}\left(u^{* *}, \phi\left(u^{* *}\right)\right)$ if $\beta=\mu$ and that $\mathcal{T}\left(u^{*}, \phi\left(u^{*}\right)\right)=\{N\}=\mathcal{T}\left(u^{* *}, \phi\left(u^{* *}\right)\right)$ if $0<\beta<\mu$. It follows that $\mathcal{T}\left(u^{* *}, \phi\left(u^{* *}\right)\right)=\mathcal{T}\left(u^{*}, \phi\left(u^{*}\right)\right)$. Thus, applying CADD yields

$$
\phi\left(u^{*}+u^{* *}\right)=\phi\left(u^{*}\right)+\phi\left(u^{* *}\right)=\alpha e_{T}-\beta e_{N \backslash T}+\lambda \frac{|N \backslash T|}{|N|} e_{N} \neq \lambda e_{N} .
$$

On the other hand, it is easily verified that $\lambda e_{N}=\operatorname{Es}\left(u^{*}+u^{* *}\right) \in C\left(u^{*}+u^{* *}\right)$, so it follows from Lemma 2 that $\phi\left(u^{*}+u^{* *}\right)=\lambda e_{N}$. Contradiction.

So, $\phi\left(u^{*}\right)=\lambda e_{T}$. By choosing $\mu$ sufficiently large $(\mu \geq \lambda \kappa)$, we obtain $u^{*} \leq$ $\lambda w^{T, \mathcal{T}, \kappa}$. Moreover, we have $\phi\left(u^{*}\right)=\lambda e_{T} \in C\left(\lambda w^{T, \mathcal{T}, \kappa}\right)$. The lemma now follows from IIC.

Lemma 5 Let $v$ be a balanced game and let $\mathcal{T}:=\mathcal{T}(v, \operatorname{LS}(v))$. Then there exist nonnegative numbers $\lambda_{T}(T \in \mathcal{T} \backslash\{N\})$ and a real number $\lambda_{N}$ such that $\operatorname{LS}(v)=$ $\sum_{T \in \mathcal{T}} \lambda_{T} e_{T}$

Proof Recall that LS $(v)$ is defined as the (unique) solution to the following minimization problem.

$$
\min \langle x, x\rangle \text { subject to } x \in C(v)
$$

Now, the Kuhn Tucker optimality conditions for this problem state that

$$
2 \mathrm{LS}(v)+\sum_{S \subseteq N} \mu_{S} e_{S}=0
$$

with $\mu_{S} \leq 0$ for all $S \neq N$ and $\mu_{S}(\operatorname{LS}(v)(S)-v(S))=0$ for all $S \subseteq N$. The claim of the Lemma follows immediately.

Lemma 6 Let $\phi$ be a core concept that satisfies IIC, ETP and CADD. Then $\phi(v+$ $\left.\lambda e_{N}\right)=\phi(v)+\lambda e_{N}$ for all balanced games $v$ and all $\lambda \in \mathbb{R}$.

Proof We may assume that $\lambda \neq 0$. We distinguish the two cases $\lambda>0$ and $\lambda<0$. If $\lambda>0$, write $\mathcal{T}:=\mathcal{T}(v, \phi(v))$ and $w:=\lambda w^{N, \mathcal{T}, 1}$. By Lemma $4, \phi(w)=\lambda e_{N}$. Therefore $\mathcal{T}(w, \phi(w))=\mathcal{T}$. Then by CADD,

$$
\phi(v+w)=\phi(v)+\phi(w)=\phi(v)+\lambda e_{N} .
$$

Moreover, $v+\lambda e_{N} \geq v+w$. Hence $C\left(v+\lambda e_{N}\right) \subseteq C(v+w)$, and

$$
\phi(v+w)=\phi(v)+\lambda e_{N} \in C\left(v+\lambda e_{N}\right) .
$$


Applying IIC, we obtain

$$
\phi\left(v+\lambda e_{N}\right)=\phi(v+w)=\phi(v)+\lambda e_{N} .
$$

Now assume that $\lambda<0$. In this case, define $u=v+\lambda e_{N}$ and apply the above reasoning to the game $u$. This yields $\phi(v)=\phi\left(u-\lambda e_{N}\right)=\phi(u)-\lambda e_{N}$. By rearranging terms we obtain

$$
\phi\left(v+\lambda e_{N}\right)=\phi(u)=\phi(v)+\lambda e_{N} .
$$

Now, we have devised enough tools to prove the main result of this section.

Theorem 4 The LS concept is the only core concept that satisfies IIC, ETP and CADD.

Proof We have shown already that LS indeed satisfies the three axioms. Let $\phi$ be a solution that satisfies IIC, ETP and CADD. We show that $\phi=\mathrm{LS}$.

Take an arbitrary balanced game $v$ and let $\mathcal{T}:=\mathcal{T}(v, \operatorname{LS}(v))$. By Lemma 5 we can write

$$
\operatorname{LS}(v)=\sum_{T \in \mathcal{T}} \lambda_{T} e_{T}
$$

with $\lambda_{T} \geq 0$ for all $T \neq N$. Define, for $\kappa>0, u^{\kappa}:=\sum_{T \in \mathcal{T} \backslash\{N\}} \lambda_{T} w^{T, \mathcal{T}, \kappa}$, and define $w^{\kappa}=u^{\kappa}+\lambda_{N} e_{N}$. By Lemma $4, \phi\left(\lambda_{T} w^{T, \mathcal{T}, \kappa}\right)=\lambda_{T} e_{T}$. From this it follows that $\mathcal{T}\left(\lambda_{T} w^{T, \mathcal{T}, \kappa}, \lambda_{T} e_{T}\right)=\mathcal{T}$ for all $T \in \mathcal{T}$ with $\lambda_{T}>0$. Thus, by CADD,

$$
\phi\left(u^{\kappa}\right)=\sum_{T \in \mathcal{T} \backslash\{N\}} \lambda_{T} e_{T} .
$$

Now Lemma 6 yields

$$
\phi\left(w^{\kappa}\right)=\phi\left(u^{\kappa}+\lambda_{N} e_{N}\right)=\phi\left(u^{\kappa}\right)+\lambda_{N} e_{N}=\sum_{T \in \mathcal{T}} \lambda_{T} e_{T}=\operatorname{LS}(v) .
$$

Finally, observe that $v \geq w^{\kappa}$ for sufficiently large $\kappa$, and that $\phi\left(w^{\kappa}\right) \in C(v)$. By taking a sufficiently large $\kappa$ it follows from IIC that $\phi(v)=\phi\left(w^{\kappa}\right)=\operatorname{LS}(v)$.

In Sect. 4 we constructed a non-egalitarian core concept that satisfies IIC and ETP according to Proposition 1. To prove independence of the axioms that determine the LS concept, we conclude this paper with two examples; a core concept that satisfies IIC and CADD but not ETP, and a core concept that satisfies CADD and ETP but not IIC.

For an arbitrary player $i \in N$, let $\delta^{i}$ denote the solution that assigns the vector $v(N) e_{i}$ to a game $v$. Clearly, the core concept $P\left(\delta^{i}\right)$ does not satisfy ETP. Since $\delta^{i}$ 
is additive, it follows from Theorem 3 that $P\left(\delta^{i}\right)$ satisfies CADD. It is also easily verified that $P\left(\delta^{i}\right)$ satisfies IIC.

It is not difficult to prove that, if $\phi$ is a solution that satisfies ETP, then also $P(\phi)$ satisfies ETP. Denote the Shapley value by $S h$. Then the core concept $P(S h)$ satisfies ETP (since $S h$ satisfies ETP) and CADD (by Theorem 3 and the fact that $S h$ is additive). It is easy to demonstrate that $P(S h)$ does not satisfy IIC.

Open Access This article is distributed under the terms of the Creative Commons Attribution Noncommercial License which permits any noncommercial use, distribution, and reproduction in any medium, provided the original author(s) and source are credited.

\section{References}

Arin J, Iñarra E (2001) Egalitarian solutions in the core. Int J Game Theory 30(2):187-193

Bondareva ON (1963) Some applications of linear programming methods to the theory of cooperative games. Probl Kibern 10:119-139

Dutta B (1990) The egalitarian solution and reduced game properties in convex games. Int J Game Theory 19:153-169

Dutta B, Ray D (1989) A concept of egalitarianism under participation constraints. Econometrica 57:615630

Dutta B, Ray D (1991) Constrained egalitarian allocations. Games Econ Behav 3:403-422

Hardy GH, Littlewood JE, Pólya G (1952) Inequalities, 2nd edn. Cambridge University Press, Cambridge Hougaard JL, Peleg B, Thorlund-Petersen L (2001) On the set of Lorenz-maximal imputations in the core of a balanced game. Int J Game Theory 30(2):147-165

Klijn F, Slikker M, Tijs S, Zarzuelo J (1998) Characterizations of the egalitarian solution for convex games. CentER discussion paper no. 9833, University of Tilburg, The Netherlands

Kohlberg E (1971) On the nucleolus of a characteristic game. SIAM J Appl Math 20(1):62-66

Moulin H (1988) Axioms of cooperative decision making. Cambridge University Press, Cambridge

Shapley LS (1967) On balanced sets and cores. Nav Res Logist Q 14:453-460

Thomson W (1994) Cooperative models of bargaining. In: Aumann R, Hart S (eds) Handbook of game theory. Elsevier, The Netherlands 